\title{
Performance Evaluation of Box Type Solar Cooker using PCM
}

\author{
Kritika, Earnest Vinay Prakash
}

\begin{abstract}
Solar energy is one of the renewable sources of energy which really finds its use in a wide variety of implications. By using solar panels or solar cooker we can harness such non exhaustible source of energy. In this paper the later one is used to cook the food by using solar insolation during day time and during night time Magnesium Nitrate Hexahydrate (PCM) is used which acts as a latent heat storage medium. Here the performance of the cooker is evaluated by determining the figure of merit as well as overall and exergy efficiency. On the basis of obtained data via experiment requisite graphs have been plotted. For the experiment purpose we have used here Aluminium pot since it has better thermal conductivity. These pots are of various capacities and are placed inside the box type solar cooker during experiment. The experiment has been conducted in the month of May when the effect of solar insolation is maximum. After doing the necessary energy analysis the used solar cooker is compared with the necessary guidelines to determine its grade.
\end{abstract}

Index Terms - Box type Solar cooker, Exergy efficiency, First figure of merit, PCM, Solar Insolation.

\section{INTRODUCTION}

Solar cooker is a simple device which is use the solar energy to cook the food. It is mainly used in boiling type cooking. It mainly works on the principle of solar insolation which harness the solar energy to cook the food. The performance of the cooker is examined by the energy analysis and various types of tests which were conducted to determine the efficiency of the cooker. Among of them load test and without load test are the important one. Since sun light is not present during night time so we have used here PCM which act as a latent heat storage medium which stores the heat energy during day time and during night time it radiates its energy and acts as a heating medium. So, the evaluation of the solar cooker will be done by thermal performance tests which includes the determination of figure of merits, exergy calculation and several performances and equipment-based test to check the overall working efficiency and effectiveness of solar cooker.

As we know that environment is getting polluted day by day by using the fossil fuels and moreover such source of

Manuscript revised June 5, 2019 and published on July 10, 2019

Kritika, PG Student Department of Mechanical Engineering, Sam Higginbottom University of Agriculture, Technology \& Sciences, Allahabad (Prayagraj), Uttar Pradesh, India.

Dr. Earnest Vinay Prakash, Assistant Professor Department of Mechanical Engineering, Sam Higginbottom University of Agriculture, Technology \& Sciences, Allahabad (Prayagraj), Uttar Pradesh, India. energy is exhaustible in nature. So, by switching to such renewable source of energy environmental pollution could be minimized. And, at the same time we would be moving towards sustainable growth of our world.

\section{EVALUATION OF THE WORKING OF THE BOX TYPE SOLAR COOKER}

\subsection{Determination of figure of merit}

Stagnation test is carried out under no-load condition to determine the value of figure of merit F1. The mathematical expression of $\mathrm{F} 1$ is as follows:

$$
\mathrm{F}_{1}=\frac{\eta}{U_{L S}}=\frac{T_{p s}-T_{a s}}{H_{S}}
$$

Where $\eta$ is the optical efficiency, defined as the fraction of the incident solar radiation, which reaches the bottom of the solar absorber plate during solar insolation. The stagnation temperature of the absorbing plate (Tps), ambient temperature (Tas) and solar insolation (Hs) are measured when a steady state is reached.

The second figure of merit is obtained under the load condition when the pot is loaded with the water content and is placed over the absorber plate inside the solar cooker for its determination. So, the F2 value is calculated by using the following equation:

$$
\mathrm{F}_{2}=\frac{F 1\left(m C_{P}\right)_{w}}{A\left(t_{2}-t_{1}\right)} \ln \left[\frac{1-\left(T_{W 1}-T_{a s}\right) / F_{1} H_{S}}{1-\left(T_{W 2}-T_{a s}\right) / F_{1} H_{S}}\right]
$$

Here the value of ambient temperature is determined which is the external temperature, along with that after an interval of 30 minutes the reading of the thermocouples are noted down. And all the requisite value is kept in the above formula to determine value of $\mathrm{F} 2$. Here, Tas $\left({ }^{\circ} \mathrm{C}\right)$ the average ambient temperature from the time period t 1 to $\mathrm{t} 2, \mathrm{Hs}\left(\mathrm{W} / \mathrm{m}^{2}\right)$ the average solar radiation from the time period t 1 to $t 2$ and $(\mathrm{mCp}) \mathrm{w}$ the product of the mass of water and specific heat $\left(\mathrm{J} / \mathrm{kg}^{\circ} \mathrm{C}\right)$.

\subsection{Energy and Exergy Efficiencies}

\subsubsection{Energy Efficiency}

The overall energy balance equation for Solar box cooker for the finite time interval can be drawn as follows:

[Energy gained by water in the vessel $]=[$ Energy supplied to water in the vessel] - [Energy lost from water in the vessel]

The output energy of the system which is the energy gained by the water kept in the aluminium pot can be calculated by using the following equation:

$$
\mathrm{Eo}=\mathrm{mCp}(\mathrm{Tw} 2-\mathrm{Tw} 1)
$$

In the above equation the necessary value of mass of water and specific heat capacity value is needed to be kept in order to determine the value of Eo. 
During the experiment we note down the value of Tw2 after 30 minutes intervals keeping the value of Tw1 constant which is the reading taken at the starting of the experiment. Along with it the reading of ambient temperature is also taken as well as the initial and the final temperature values also play the role in deciding the efficiency of the system, and this type of qualitative effect is placed in the above equation in order to determine its value.

The input energy (Ei) of the system which is the energy given to the water present in the aluminium pot by the help of solar insolation and by using the equation given below its value can be determined.

$$
\mathrm{Ei}=\mathrm{HsA} \Delta \mathrm{t}
$$

Where the value of solar insolation can be obtained using the solarimeter which gives the $\mathrm{Hs}$ value at the place of experiment. We also calculate the value of $\mathrm{A}$ which is the absorber area of solar cooker in the given time interval.

Thus, the energy efficiency of the solar cooker is determined by using the following equation which is basically the ratio of energy output to energy input. And, its equation is as follows

$$
\eta=\frac{\text { energy output }}{\text { energy input }}=\frac{E_{0}}{E_{i}}=\frac{\left[m_{w} C_{p w}\left(T_{w f}-T_{w i}\right)\right] / t}{I_{t} A_{s c}}
$$

\subsubsection{Exergy Efficiency}

The overall energy balance equation for exergy efficiency for the finite time interval can be drawn as follows

[Output Exergy from SBC] = [Input Exergy to SBC] [Exergy lost from SBC]

The energy input of the solar cooker for the given solar insolation can be determined from the following equation. For which the solar insolation value along with absorber plate area is determined

$$
E x i=H s\left[1+\frac{1}{3}\left(\frac{T_{o}}{T}\right)^{4}-\frac{4}{3} \frac{T_{o}}{T}\right] A \Delta t
$$

Where, Ts is the temperature of the sun which is used in the calculation for the given spectral distribution. Along with it the value of absorber plate area "A", with the initial temperature reading noted down using the thermocouple is also used in the above equation.

Also, the output exergy (Exo) of the solar cooker which is the energy gained by the water kept in the aluminium pot is also calculated and its equation is given as follows:

$$
\mathrm{E}_{\mathrm{xo}}=\mathrm{E}_{\mathrm{o}}-\mathrm{mC}_{\mathrm{p}} \mathrm{T}_{\mathrm{a}} \ln \frac{T w 2}{T w 1}
$$

The exergy efficiency ( $\mathrm{nx}$ ) of the system can be obtained using the given equation as below, which includes the known value of all the mentioned terms like mass of water, area of absorber plate etc.

$$
\eta x=\frac{m_{w} C_{p w}\left[\left(T_{w f}-T_{w i}\right)-T_{o} \ln \frac{T_{w f}}{T_{w i}}\right]}{I_{t}\left[1-\frac{4}{3} \frac{T_{a}}{T_{s}}\right] A_{s c}}
$$

\section{EXPERIMENTAL SETUP \&PROCEDURE}

The experiment with the PCM has been performed with the box type solar cooker in the month of May in the Solar Energy Lab of Mechanical Engg Department in the SHUATS
University, Prayagraj UP. The experimental setup consists of a box type solar cooker which is made using GI sheet and is insulated using Styrofoam whose thermal conductivity is $0.033 \mathrm{~W} / \mathrm{m}-\mathrm{K}$ and is its outer surface is of dimension $57.5 \mathrm{~cm}$ $\mathrm{x} 57.5 \mathrm{~cm}$ with height $17.5 \mathrm{~cm}$ and is painted black for better thermal conductivity. Now, the inner absorber plate is of dimension $47.5 \mathrm{~cm} \times 47.5 \mathrm{~cm}$ with height of $8.75 \mathrm{~cm}$ as shown in the following figure below.

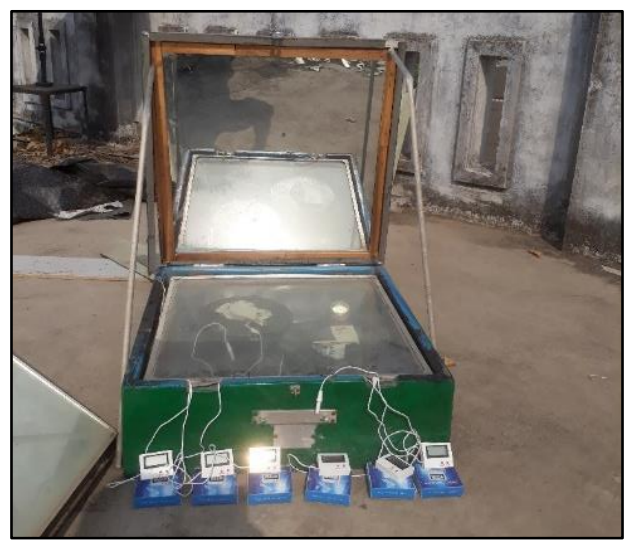

Figure 1: - Box-type Solar Cooker

Here, three Aluminium pots have been used of capacities $1.5 \mathrm{~L}$ and $0.75 \mathrm{~L}$. Two of them have large capacity such that the smaller one could be placed inside them for the experiment. Here, 6 thermocouples are used for taking the temperature readings which includes ambient temperature, absorber plate temp, PCM temp, Pot temperature etc.

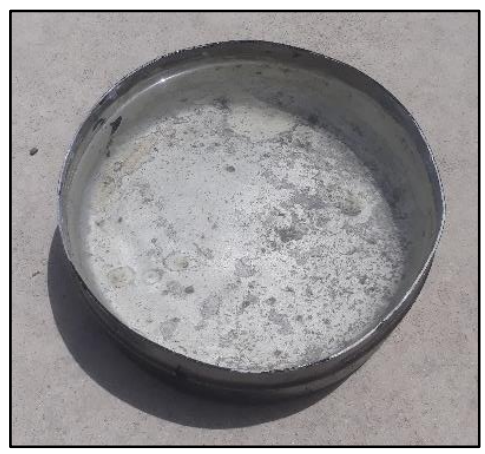

Figure 2: - Aluminium pot filled with water

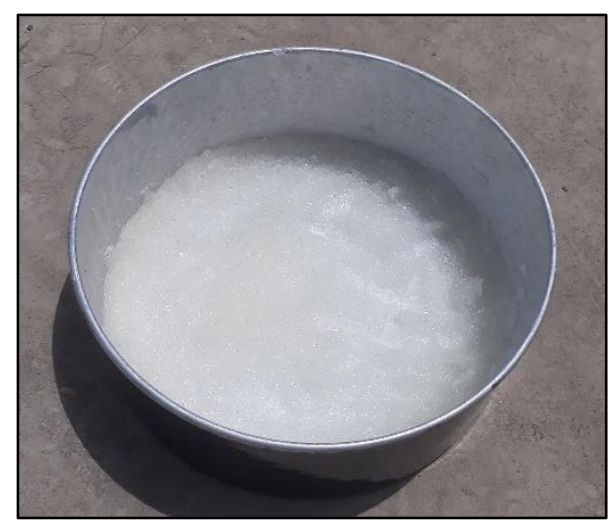

Figure 3: - PCM filled in Aluminium Pot

For PCM we have used here Magnesium Nitrate Hexahydrate which is being applied over the bottom surface of the pot which after absorption of heat changes its phase from solid to 
liquid state. Now readings are taken in an interval of 30 minutes from $9 \mathrm{am}$ to $5 \mathrm{pm}$ during day time. And, if experiment is done with PCM then the night time reading is also taken till the morning since PCM radiates its energy for night cooking.

The same set of experiment is repeated with load condition and without load condition to determine the overall and exergy efficiency of the solar cooker along with calculation of figure of merit value F1 \& F2. So, the calculated value of all the above helps us to draw a conclusion that the solar cooker falls in which grade category according to SEC guidelines and BIS.

\section{RESULT AND DISCUSSIONS}

Experiment has been done on box type solar cooker with aluminium pot material and based on the energy and energy analysis several qualitative analyses has been done. Figure 4 shows the variation of solar intensity with respect to the time of the day. Solar intensity starts increasing at steady rate before noon till it reaches the maximum, and then rate of decrease is fast. Maximum value of $920 \mathrm{~W} / \mathrm{m} 2$ is obtained at around 2 PM.

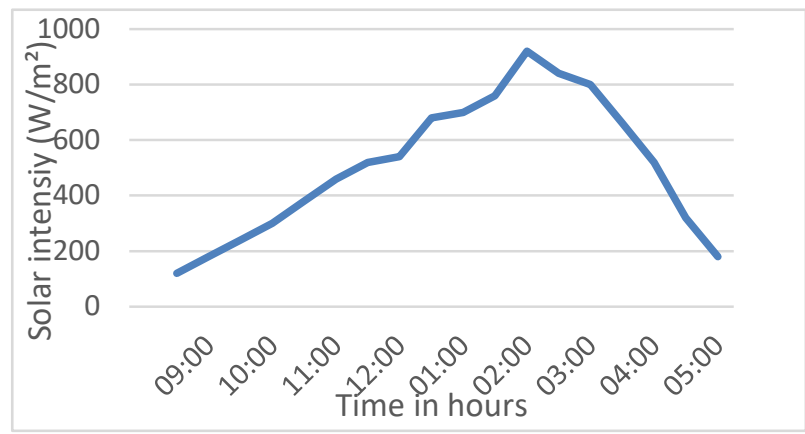

Figure 4: - Variation of solar intensity with time

Figure 5 and 6 shows the time- temperature chart for aluminum pot material together with and without load condition. So, from the Figure 5 and 6 we can infer the variation of ambient temperature, cavity temp and aluminium pot temperature with time. According to (Mullick et al., 1987 ) the figure of merit $F_{1}$ should vary in the range of 0.12

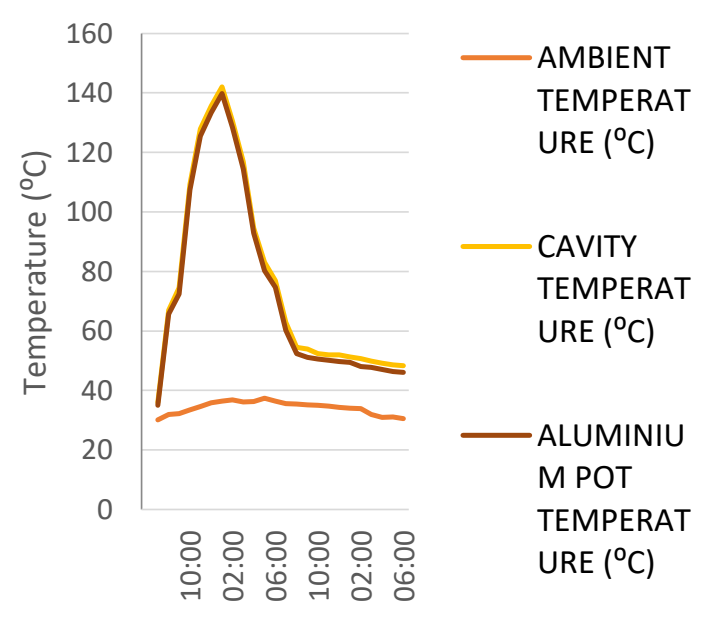

Figure 5: - Variation of temperature of cooker components without load to 0.16 . So, a higher value of $F_{1}$ indicates good efficiency and a low heat loss factor. So, the solar cooker falls in the category of Grade A, and a much higher value means cooker

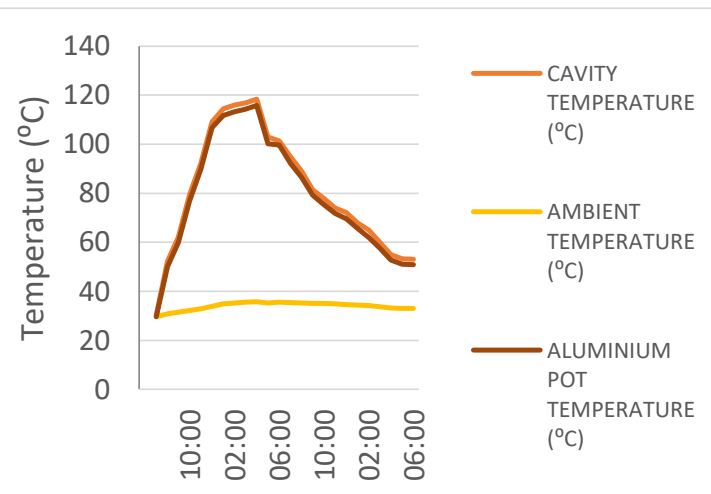

Figure 6: - Variation of temperature of cooker components with load

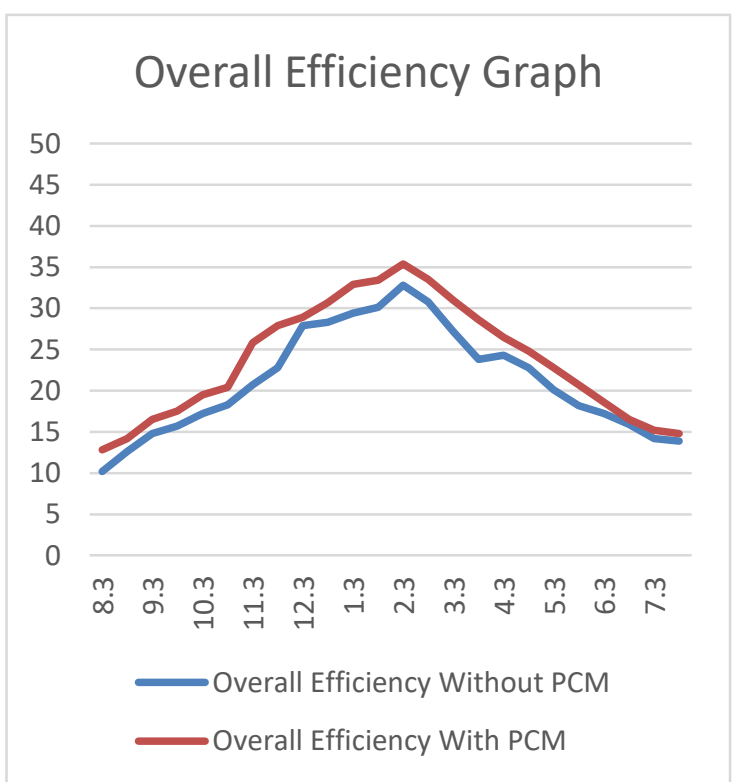

Figure 7: - Comparison of Overall Efficiency Graph

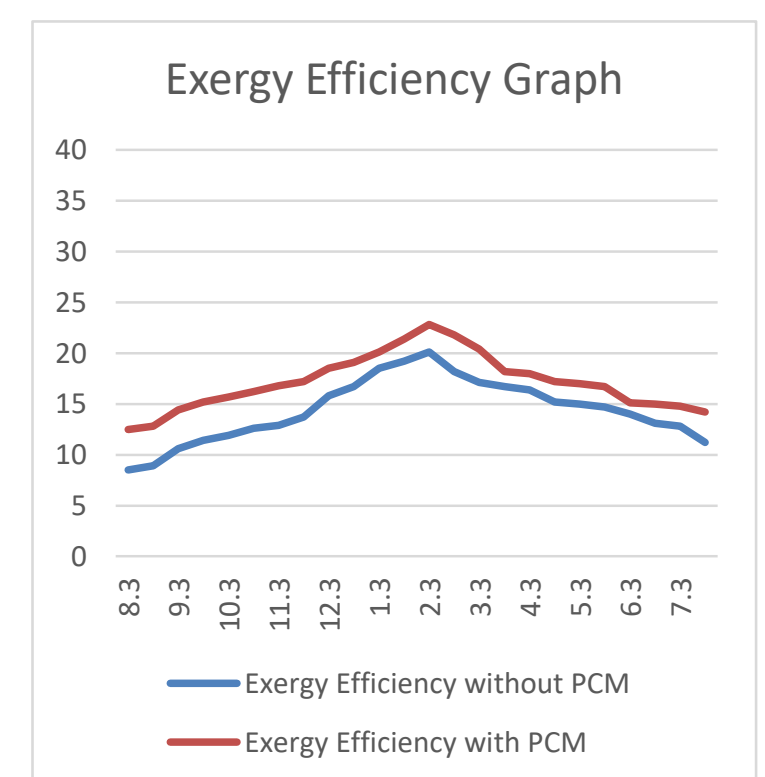

Figure 8: - Comparison of Exergy Efficiency Graph 
falling in the category of either B or C Grade. So, from the stagnation test, for aluminium pot material the calculated figure of merit $F_{1}$ is calculated as 0.126665 and the second Figure of merit $F_{2}$ for aluminium pot is 0.72194 . Finally, the results obtained using the international cooking test procedure clearly shows that the box type solar cooker used in the experiment satisfies the given set of standards.

The energy and exergy efficiencies were calculated using given equation. Energy efficiency of solar cooker with aluminium pot is $31.37 \%$. The exergy efficiency of solar cooker with aluminium pot is $21.08 \%$.

Shown in the above figure $7 \& 8$ are the comparative graph between Exergy Efficiency \& Overall Efficiency for the two conditions which are with and without the use of PCM.

\section{CONCLUSION}

In the present work, solar cooker performance is tested for the Aluminium pot material under the climate conditions of Allahabad, India for both with and without PCM. Following conclusion have been drawn:

The first figure of merit $(\mathrm{F} 1)$ is found as 0.126665 for box-type solar cooker with aluminium pots. According to BIS, solar cooker falls in A Grade category.

The second figure of merit (F2) for solar cooker with aluminium pots is 0.72194 . So, both of these values are well within the prescribed values as suggested by IS code 13429-1 (2000).

According to the results it is inferred that aluminium pot is suitable for boiling type cooking. Aluminium pot is used to fast cooking in day time cooking. Energy efficiency of solar cooker with aluminium pot is $31.37 \%$. The exergy efficiency of solar cooker with aluminium pot is $21.08 \%$.

\section{REFERENCES}

[1] Mullick, S. C., Kandpal, T. C. and Saxena A. K. Thermal test procedure for box type solar cooker. Solar Energy, 39, 1987, pp. 353360 .

\section{AUTHORS PROFILE}

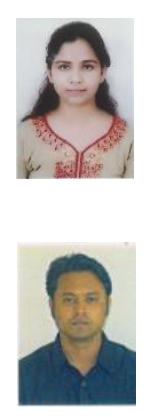

Kritika has accomplished B.Tech. from United College of Engineering \& Research Allahabad (Affiliated to AKTU), and M.Tech from SHUATS Prayagraj. Her area of interest is Thermal Engineering. Kritika had worked as a Guest Faculty at IERT Prayagraj for few years. She has attained many seminars and conferences.

Dr. Earnest Vinay Prakash has accomplished B.E. from R.G.T.U. Jabalpur, M.Tech and PhD from SHUATS Prayagraj and presently associated as Assistant Professor at SHUATS, U.P. India. He has published many research papers in national / international journals. Dr. Vinay has attained many conferences and seminar.
[2] Funk, P. A. and Larson, D. L. Parametric model of solar cooker performance. Solar Energy, 62(1), 1998, pp. 63-8.

[3] Funk, P. A. Evaluating the international standard procedure for testing solar cookers and reporting performance. Solar Energy, 68(1), 2000, pp. 1-7.

[4] Harmim, A., Belhamel, M., Boukar, M. and Amar, M. Experimental investigation of boxtype solar cooker with a finned absorber plate. Energy, 35, 2010, pp. 3799-3802.

[5] Kumar, N., Agravat, S., Chavda, T. and Mistry, H. N. Design and development of efficient multipurpose domestic solar cookers/ dryers. Renewable Energy, 33, 2008, pp. 2207-2211.

[6] Ozturk, H. H. Comparision of energy and exergy efficiency for solar box and parabolic cookers. J Energy Engg, 133(1), 2007, pp. 53-62.

[7] Petela, R. Exergy analysis of the solar cylindrical-parabolic cooker. Solar Energy, 79, 2005, pp. 221-233.

[8] Kumar, N., Vishwanath, G. and Gupta, A. Anexergy based test protocol for truncated pyramid type solar box cooker. Energy, 36, 2011, pp. 5710-5715.

[9] Algiri A.H and Towale, H.A (2001): Efficient orientation impacts of box type solar cookers on the cooker performance. Solar Energy 70,165-170

[10] Ammer E.H, (2003): Theoretical and experimental assessment of double exposure solar cooker, Energy Conservation and Management 44, $2034-2043$

[11] Arezki H. et al (2008). Experimental study of double exposure solar cooker with finned cooking Vessel. Solar Energy 82,287-289

[12] Dasin D.Y, Habou D, Rikoto II, (2011), Performance evaluation of parabolic solar concentrator against international standard procedure in the tropical Environment. Nigerian Journal of Renewable Energy, 15, 20-28.

[13] BIS (1992). Indian Standard-Solar Cooker, IS 13429:1992, Bureau of Indian Standards, Manak Bhawan, New Delhi- 110002. 